\title{
Erratic Dislocations within Funnel Defects in AlN Templates for AlGaN Epitaxial Layer Growth
}

\author{
M. E. Hawkridge ${ }^{1, \text { a) }}$, Z. Liliental-Weber ${ }^{1}$, H. J. Kim², S. Choi², D. Yoo ${ }^{2}$, J. -H. Ryou ${ }^{2}$, \\ and R. D. Dupuis ${ }^{2}$
}

${ }^{I}$ Materials Science Division, Lawrence Berkeley National Laboratory, 1 Cyclotron Road MS 62R0209/213, Berkeley, CA 94720, USA

${ }^{2}$ Center for Compound Semiconductors and School of Electrical and Computer Engineering, Georgia Institute of Technology, 777 Atlantic Dr. NW, Atlanta, Georgia 30332-0250, USA

\begin{abstract}
We report our transmission electron microscopy observations of erratic dislocation behavior within funnel-like defects in the top of AlN templates filled with $\mathrm{AlGaN}$ from an overlying epitaxial layer. This dislocation behavior is observed in material where phase separation is also observed. Several bare AlN templates were examined to determine the formation mechanism of the funnels. Our results suggest that they are formed prior to epitaxial layer deposition due to the presence of impurities during template re-growth. We discuss the erratic dislocation behavior in relation to the
\end{abstract}


presence of the phase-separated material and the possible effects of these defects on the optoelectronic properties.

a) Author to whom correspondence should be addressed. Electronic mail: mehawkridge@1bl.gov 
Alloys of $\mathrm{AlGaN}$ are of great interest for optoelectronic devices that operate in the UV region of the electromagnetic spectrum due to their direct tunable band gaps in this energy range ${ }^{1}$. High Al-content alloys are of particular interest for deep-UV applications such as light emitting diodes (LEDs), photodetectors, and bio-purification ${ }^{2}$. Due to the relative lack of native substrates, these alloys are usually grown by heteroepitaxial techniques on substrates such as sapphire, which introduces high densities of defects and strain into the epitaxial layer detrimental to the final quality. This mismatch can be relieved somewhat by employing a thick template material such as $\mathrm{GaN}$, but growth of Al-rich alloys on $\mathrm{GaN}$ introduces significant tensile strain into the layer that can cause cracking ${ }^{3}$. AlN templates are therefore preferred for Al-rich AlGaN alloys and have been utilized recently to grow high-quality UV-LED structures ${ }^{4}$. As AIN templates become commercially available, they will see greater use for such devices. However, the preparation and quality of the AlN template is critical to obtaining high quality epitaxial layers and is often glossed over in the drive to obtain working devices.

In addition to the issues surrounding heteroepitaxy, growth of high $\mathrm{Al}$ content AlGaN alloys by conventional metalorganic chemical vapor deposition (MOCVD) is hindered by the high sticking coefficient of $\mathrm{Al}$ compared to $\mathrm{Ga}$ adatoms and a parasitic gas-phase reaction of TMAl and ammonia ${ }^{2,5}$. One of the growth methods available for AlGaN that avoids the need for high growth temperatures ${ }^{6-8}$ is the modulated precursor epitaxial growth (MPEG). In this growth scheme, the group III and V precursors are introduced into the growth chamber separately in an alternating sequence, reducing the parasitic gas-phase reactions and increasing the adatom migration ${ }^{9,10}$. 
In this paper, we report our transmission electron microscopy (TEM) observations of unusual funnel-like defects and erratic dislocations in the surface of AlN templates used for $\mathrm{Al}_{\mathrm{x}} \mathrm{Ga}_{1-\mathrm{x}} \mathrm{N}$ growth by MPEG. Further analysis of conventionally grown bare AlN templates indicates that the funnels are pre-existing hollow defects filled in by the epitaxial material. We discuss the possible formation mechanisms for these funnels and the associated dislocation behavior.

AlN templates were grown on (0001) sapphire substrates using a typical two-step growth of heteroepitaxy employing a low-temperature AlN buffer layer by conventional metalorganic vapor phase epitaxy (MOCVD) to a thickness of around $1 \mu \mathrm{m}$. The total dislocation density for the templates was considered to be high (estimated to be on the order of $10^{9} \mathrm{~cm}^{-2}$ of magnitude), while the surface was confirmed to be free of nanopits (or V-defects) by atomic force microscopy (AFM). $\mathrm{Al}_{\mathrm{x}} \mathrm{Ga}_{1-\mathrm{x}} \mathrm{N}$ layers of varying composition $(0.6<\mathrm{x}<1.0)$ were grown on these templates by MOCVD in the MPEG regime following a brief period $(\sim 200 \mathrm{~nm})$ of conventional AlN template re-growth. The composition of the AlGaN layer was controlled by the ratio of the precursors used in the group-III stage of growth (further details are provided in Ref. 10). Cross-sectional TEM specimens were prepared using standard polishing and ion milling techniques. Conventional TEM images were obtained using a JEOL 3010 TEM and Z-contrast TEM images were obtained using a Philips G2 Tecnai operating at 200kV.

Standard TEM characterization of several AlGaN layers showed that the layer qualities were very good in general. The samples with higher $(\mathrm{x}>0.75)$ aluminum content had smooth surfaces and good crystal perfection indicated by selected area diffraction. In most cases, the interface with the template was observed to be uniformly 
smooth and threading dislocations in the template propagated into the epitaxial layer with minimal interaction. However, some samples exhibited unusual defects in the surface of the template and less than ideal $\mathrm{Al}_{\mathrm{x}} \mathrm{Ga}_{1-\mathrm{x}} \mathrm{N}$ layer qualities for $\mathrm{x}<0.75$.

Fig. 1 shows bright field TEM images of the epitaxial $\mathrm{Al}_{\mathrm{x}} \mathrm{Ga}_{1-\mathrm{x}} \mathrm{N}$ layer in a sample with $\mathrm{x} \sim 0.6$. The surface of the AlGaN layer is relatively rough, with approximately $\pm 20 \mathrm{~nm}$ thickness variation throughout the sample. In the image taken under $\mathrm{g}=(0006)$ conditions [Fig. 1(a)], dislocations with a screw component are observed to thread through the template and into the AlGaN layer well aligned with the [0001] direction. This is considered typical dislocation behavior for epitaxial layer growth. However, some of the dislocations appear to have a highly erratic path close to the interface with the epitaxial layer in the upper $\sim 150 \mathrm{~nm}$ of the template. The almost helical path in some cases is very unusual, as this orientation does not minimize the line tension of the dislocation. When compared to an image taken under kinematical diffraction imaging conditions [Fig. 1(b)], i.e. away from any strongly diffracting Bragg reflection such that mass-thickness is the dominant contrast mechanism, it becomes apparent that the erratic dislocation behavior occurs within the boundaries of funnel-shaped defects in the template layer. The funnels in Fig. 1(b) appear darker than the surrounding AlN material, suggesting that they are filled with material from the overlying AlGaN layer (containing elements with higher atomic number Z).

The filling of the funnels is more clearly shown in high angle annular dark field (z-contrast) in Fig. 2. It may also be seen from this image that the AlGaN layer has undergone phase-separation, exhibiting bright and dark regions corresponding to Ga- and Al-rich material, respectively. Indeed, closer examination shows that some of the funnels 
are filled with phase-separated material. The phase separation occurs here due to the limited incorporation of gallium in the absence of an ammonia overpressure in the MPEG regime and contributes to the roughness of the layer ${ }^{10}$. Similar observations have been made in conventionally grown AlGaN material, where phase separation or atomic ordering occurs to accommodate the differences in tetrahedral radii between $\mathrm{Al}$ and $\mathrm{Ga}$ atoms $^{11,12}$.

To gain more insight into the formation of these defects, a range of bare AIN template samples grown at different temperatures were studied. In general, AlN layers grown at lower temperatures $\left(c .1050^{\circ} \mathrm{C}\right.$ ) exhibited more surface pits in AFM scans compared to those grown at higher temperatures (c. $\left.1100^{\circ} \mathrm{C}\right)$. Standard TEM images of such samples are shown in Fig. 3, taken under kinematical diffraction imaging conditions. In the samples studied, hollow nanopipes are observed to open around the depth corresponding to the re-growth interface. In the layer grown at $1140^{\circ} \mathrm{C}$ [Fig. 3(a)], the majority of these nanopipes are all fully constricted before the surface. However, many nanopipes remain open to the surface of the layer grown at $1060^{\circ} \mathrm{C}$ [Fig. 3(b)], dilating in the last $\sim 50 \mathrm{~nm}$ to form the funnel-like shape. By imaging in orthogonal $\mathrm{g}=$ (0006) and $g=(11-20)$ conditions and using the invisibility criteria, it was determined that the nanopipes and funnels appear to be associated with screw and mixed-type dislocations.

These results clearly indicate that the funnel defects are formed prior to the deposition of the AlGaN layer from nanopipes that are open to the surface of the AlN template. The formation of nanopipes in $\mathrm{GaN}$ has been related to the presence of impurities such as oxygen ${ }^{13-15}$, which can be present in MOCVD grown material. This 
effect may be greater in AlN due to the increased affinity of Al to oxidize compared to Ga. Indeed, the template may have an oxide layer on its surface (despite cleaning efforts) that acts as an impurity source during re-growth of the template. This would explain why the nanopipes/ funnels all open at a depth corresponding to the nominal re-growth layer thickness in Fig. 3. At the higher bare AlN layer growth temperature, the affinity of Al to oxidize may be reduced and the sticking coefficient of oxygen adatoms is decreased, allowing the impurities to be driven off and the nanopipes to close before the end of growth (Also note the tapering shape of some of the nanopipes in Fig. 3 as the impurities are driven off). At the lower growth temperature, it is the opposite case and the nanopipes remain open to the surface of the layer. Where this occurs, the dilation of the nanopipe at the surface most likely occurs due to a relaxation of the strain field surrounding the dislocation or some kinetic effect related to the presence of impurities on inclined planes ${ }^{16}$, forming the funnel shape. It should be noted that this argument suggests growth for longer periods may allow any remaining nanopipes to close off as any remaining impurity sources are exhausted. Indeed, the templates used for the MPEG samples were only re-grown by $\sim 200 \mathrm{~nm}$ compared to the $\sim 500 \mathrm{~nm}$ of the bare AlN templates in Fig. 3, increasing the likelihood of observing these funnels.

Any hollow funnels in the template layer are likely to be filled-in during subsequent growth. The dislocation that terminates at the base of the hollow funnel (as this is the effective surface of the template) is then propagated into the epitaxial layer and into the filled funnel. A similar process has been observed for V-pits in $\mathrm{InGaN} / \mathrm{GaN}$ multi quantum wells (MQWs), where it should be noted that the deformation in the MQW structure leads to localization of charge carriers ${ }^{17}, 18$. In the case of the MPEG 
sample shown in Figs. 1 and 2, the material filling the funnels undergoes phase separation and the path of the dislocation becomes extremely erratic as a result. The presence of the phase-separated material will act to localize carriers within the confines of the funnel. However, dislocations are known to be sites of non-radiative recombination in $\mathrm{GaN}^{19}$, which would counteract this localization. Lastly, if such funnel defects were to form in a heterostructure, they could act as shortage path in a manner similar to that observed by Hsu et al in $\mathrm{GaN}^{20}$.

We examined several AlGaN layers grown by MOCVD in the MPEG regime on AlN templates using TEM techniques. Unusual funnel-like defects were observed in the top of some AlN templates that were filled with $\mathrm{Al}_{\mathrm{x}} \mathrm{Ga}_{1-\mathrm{x}} \mathrm{N}$ material from the overlying layer. Erratic dislocation paths were formed within some of the funnels in the $\sim 150 \mathrm{~nm}$ nearest the template surface as a result of phase separation in the ternary AlGaN layer. Studies of bare AIN templates revealed that the funnels were pre-existing hollow defects. We believe that they are formed due to the presence of impurities such as oxygen, which is consistent with the funnels being less common at higher growth temperatures. Our results also indicate that growth for longer periods may reduce the probability that these defects are formed.

\section{Acknowledgements}

This work was supported by the Georgia Institute of Technology contract R7776S2 (under the DARPA DUVAP Program Contract FA8718-07-C-0002) through the U.S. Department of Energy under Contract No. DE-AC02-05CH11231 and was 
performed at the National Center for Electron Microscopy, Lawrence Berkeley National Laboratory. 


\section{References}

${ }^{1}$ H. Morkoc, Nitride Semi-Conductors and Devices, Springer-Verlag, Berlin (1999).

${ }^{2}$ M. A. Khan, M. Shatalov, H. P. Maruska, H. M. Wang, and E.Kuokstis: Jpn. J. Appl. Phys. 44, 7191 (2005).

${ }^{3}$ S. Einfeldt, M. Diesselberg, H. Heinke, D. Hommel, D. Rudloff, J. Christen, and R. F. Davis, J. Appl. Phys. 92, 118 (2002).

${ }^{4}$ S. Sumiya, Y. Zhu, J. Zhang, K. Kosaka, M. Miyoshi, T. Shibata, M. Tanaka, and T. Egawa, Jpn. J. Appl. Phys. 47, 43 (2008).

${ }^{5}$ C. H. Chen, H. Liu, D. Steigerwald, W. Imler, C. P. Kuo, M. G. Craford, M. Ludowise, S. Lester, and J. Amano, J. Electron. Mater. 25, 1004 (1996).

${ }^{6}$ Y. Ohba and A. Hatano, Jpn. J. Appl. Phys. 35, L1013 (1996).

${ }^{7}$ M. Imura, K. Nakano, T. Kitano, N. Fujimoto, N. Okada, K. Balakrishnan, M. Iwaya, S. Kamiyama, H. Amano, I. Akasaki, K. Shimono, T. Noro, T. Takagi, and A. Bandoh, Phys. Stat. Sol. (a) 203, 1626 (2006).

${ }^{8}$ J. Han, J. J. Figiel, M. H. Crawford, M. A. Banas, M. E. Bartram, R. M. Biefeld, Y. K. Song, and A. V. Nurmikko, J. Crystal Growth 195, 291 (1998).

${ }^{9}$ H. J. Kim, S. Choi, D. Yoo, J.-H. Ryou, R. D. Dupuis, R. F. Dalmau, P. Lu, and Z. Sitar, Appl. Phys. Lett. 93, 022103 (2008).

${ }^{10}$ H. J. Kim, S. Choi, D. Yoo, J.-H. Ryou, and R. D. Dupuis, J. Crystal Growth 310, 4880-4884 (2008)

${ }^{11}$ A. Wise, R. Nandivada, B. Strawbridge, R. Carpenter, N. Newman, and S. Mahajan, Appl. Phys. Lett. 92, 261914 (2008). 
${ }^{12}$ A.T. Wise, D.-W. Kim, N. Newman and S. Mahajan, Scripta Materialia 54, 153-157 (2006)

${ }^{13}$ M. E. Hawkridge and D. Cherns, Appl. Phys. Lett. 87, 221903 (2005).

${ }^{14}$ I. Arslan and N. D. Browning, Phys. Rev. Lett. 91,165501(2003).

${ }^{15}$ J. Northrup, Phys. Rev. B 73, 115304 (2006).

${ }^{16}$ Z. Liliental-Weber, Y. Chen, S. Ruvimov and J. Washburn, Phys. Rev. Lett. 79, 2835 (1997).

${ }^{17}$ Z. Liliental-Weber, J. of Electron Microscopy 49, 339-348 (2000)

${ }^{18}$ A. Hangleiter, F. Hitzel, C. Netzel, D. Fuhrmann, U. Rossow, G. Ade and P. Hinze, Phys. Rev. Lett. 95, 127402 (2005).

${ }^{19}$ E. Müller, D. Gerthsen, P. Brückner, F. Scholz, Th. Gruber and A. Waag, Phys. Rev. B 73, $245316(2006)$.

${ }^{20}$ J. W. P. Hsu, M. J. Manfra, S. N. G. Chu, C. H. Chen, L. N. Pfeiffer and R. J. Molnar, Appl. Phys. Lett. 78, 3980 (2001). 
Figure 1: (a) Bright field two beam image of AlGaN layer $(\mathrm{x} \sim 0.6)$ grown by MPEG on AlN template. Erratic dislocation behavior is observed near the top of the template. (b) Kinematical image of the same area showing that the erratic dislocations occur within the boundaries of funnel-like defects. These funnels are filled, as they appear darker in comparison to the surrounding AlN layer.

Figure 2: (a) Z-contrast image of showing the presence of phase-separated material in the AlGaN layer and within the funnels (arrowed).

Figure 3: TEM images taken away from any strongly diffracting condition of AlN layers grown on AlN templates at (a) $1140^{\circ} \mathrm{C}$ and (b) $1060^{\circ} \mathrm{C}$. The arrows mark the approximate position of the interface between the AlN template and regrown AlN. The surface of the $1140^{\circ} \mathrm{C}$ layer is smooth while the $1060^{\circ} \mathrm{C}$ sample surface exhibits hollow funnel-like defects that extend several hundreds of nanometers into the layer. 


\section{AIGaN}

\section{AlGaN}

\section{AIN}

$50 \mathrm{ng}$ (b)
$50 \mathrm{~nm}$ 
Ga-rich

AIN 


\section{Regrown AIN}

(a)
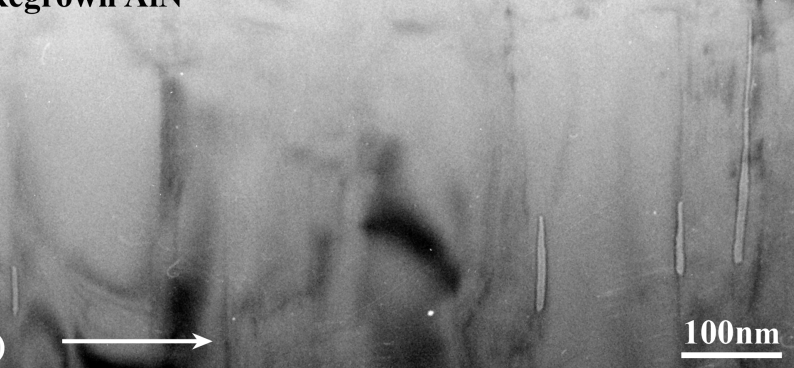

Regrown AIN

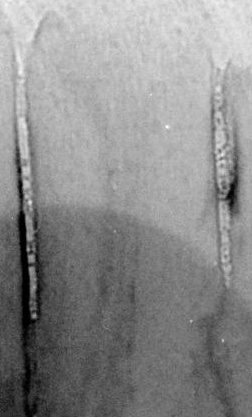

$100 \mathrm{~nm}$

(b) 